\title{
Optimization of the Passenger Throughput at an Airport Security Checkpoint Based on Queuing Theory
}

\author{
Wuyu Zhang ${ }^{1, a}$ \\ ${ }^{1}$ School of Electrical \& Electronic Engineering, North China Electric Power University, Baoding \\ 071000, China \\ azwy5432@163.com
}

Keywords: queuing theory, airport security, bottleneck, optimization.

\begin{abstract}
Airport security is an integral part of the national transportation infrastructure and a critical aspect of airport operations globally. In order to reduce the length of stay in the terminal, it is necessary to optimize the security services. I take the O'Hare Airport as the research object. Firstly, I build a queuing theory model to calculate the time spent on each process in Airport Security Checkpoint and define the efficiency of the security system. Then, I analyze the flow of passengers through a security check point baes on the model, and I find that one of the bottlenecks is that passengers are waiting too long for packaging baggage. The other one is the waste of resources because the time passengers through the mm wave body scanner is greater than that the luggage through the X-ray machine.
\end{abstract}

\section{Introduction}

Airport security is an integral part of the national transportation infrastructure and a critical aspect of airport operations globally. According to the TSA, over 600 million passengers travel on commercial airlines and more than 700 million pieces of luggage are screened each year. After 9/11, airport security has been significantly enhanced throughout the world. Airports have security checkpoints, increasing the security at the price of inconvenience and timely delays. The current process for a US airport security checkpoint is displayed in Fig.1.

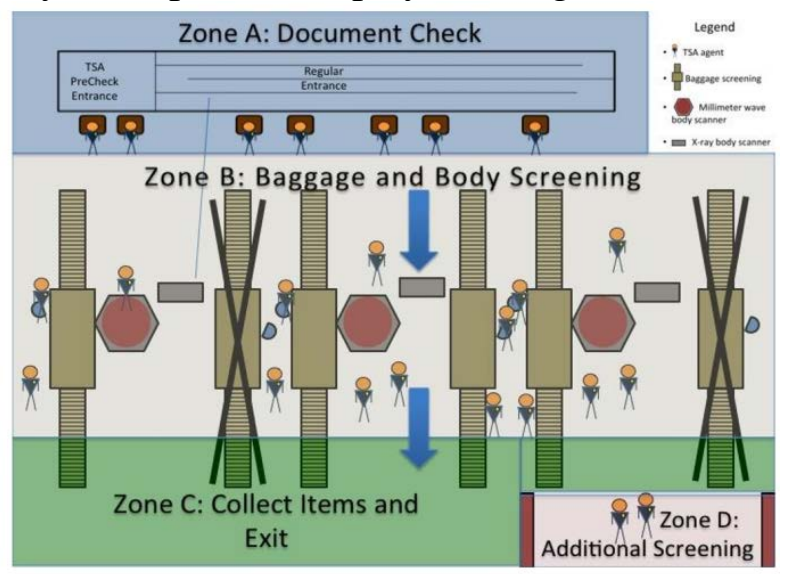

Fig. 1 Illustration of the TSA Security Screening Process.

In order to reduce the length of stay in the terminal, it is necessary to optimize the security services. A new program is required to review airport security checkpoints and staffing to identify potential bottlenecks that disrupt passenger throughput. The program should both increase checkpoint throughput and reduce variance in wait time, all while maintaining the same standards of safety and security. So it needs to solve such a problem: build a model to explore passenger flow through security checkpoints and identify bottlenecks.

\section{Queuing Theory Model}

A queuing theory model is built to calculate the time spent on each process in Airport Security Checkpoint and define the efficiency of the security system. In this model, considering the general 
situation, because of the small sample data capacity, $\mathrm{M}$ / $\mathrm{M}$ / N queuing system is given, that is, the passengers arrive at the airport security zone accord with the Poisson flow, so the passenger arrival time interval obeys negative exponential distribution. The case process in the security zone is a birth and death process. If $\mathrm{N}(\mathrm{t})$ is the number of passengers in the system at time $\mathrm{t}$, then $\{\mathrm{N}(\mathrm{t}), t \geq 0\}$ can constitute a special random process, its probability distribution has the following properties ${ }^{[1]}$ :

- Let $N(t)=n$, then the time from t to the next customer arrival time follows the negative exponential distribution of the parameter $\lambda_{n}(\mathrm{n}=0,1,2 \cdots)$;

- Let $N(t)=n$, then the time from t to the next passenger departure time follows the negative exponential distribution of the parameter $\mu_{n}(\mathrm{n}=0,1,2 \cdots)$;

- In a sufficiently small time interval $(t, t+\Delta t)$, the probability of two or more passengers arriving or leaving, that is, only one passenger arrives or leaves at the same time.

In these rules, $\lambda_{n}$ is the average arrival rate of the passengers in t time, $\mu_{n}$ is the number of passengers completed security screening per unit time, is the service rate. From the process of security screening, a passenger should at least go through three zones when accepting security screening. According to the actual situation, for these three models, the following assumptions should be made: Passengers passed the zone A all enter the zone B to accept security screening. That is, the service rate of zone $A$ is the arrival rate of zone $B$. Some of the passengers passed the zone $\mathrm{B}$ enter the zone $\mathrm{D}$ to accept the further inspection, and the others all enter the zone $\mathrm{C}$ to pick up baggage and leave. The id-check time of pre-check and regular passengers in the zone A is approximately equal. At the same time, according to the passenger traffic of airport in one day, it can regard the 8:00-9:00, 13:00-16:00 and 18:00-20:00 as the three peaks of security screening and regard the other periods from 7:20-20:00 as the stationary period, the period from 21.00 to 7:00 of the next day as the trough period. Queuing model is established for the security screening system at peak times.

\subsection{Model Assumption}

- I assume that the efficiency of security inspectors is the same.

- I assume that checking machine is working properly, without any trouble.

- I assume that passengers who have finished the security screening in the zone A go to the zone $\mathrm{B}$ to accept security screening immediately, the middle time is ignored. From zone B to zone $\mathrm{C}$ is the same.

- Zone B, D can be unlimited queuing.

- In the zone B, a service desk only allows a person to pass at a certain moment.

2.2 Symbol Definitions

Table 1 Symbols of queuing model

Symbol Definition

\begin{tabular}{l|r}
$\alpha$ & the efficiency of the security zone \\
$\rho$ & security service intensity \\
$L_{s}$ & the total number of passengers in the security zone \\
$W_{s}$ & expected value of passenger stay time in security screening system \\
$L_{q}$ & the number of passengers waiting for security screening in security zone \\
$W_{q}$ & the expected value of the passengers' waiting time in the queue of security zone \\
$\lambda_{n}$ & average number of passengers arriving at airport per unit of time in security zone \\
$\mu_{n}$ & the number of passengers who can complete the security screening per unit of \\
& time in the security zone
\end{tabular}

\subsection{Model Solving}

First, the id-check time of pre-check and regular passengers in the zone A is approximately equal. Assuming that there are $l$ service desk conducting id-check, then its average arrival rate $\lambda$ and average service rate $\mu$ can be determined by the passenger's traffic of the airport ${ }^{[2]}$.

$$
\lambda_{A}=\frac{X}{T_{a}}
$$




$$
\mu_{A}=\frac{X^{\prime}}{T_{a}^{\prime}}
$$

In the formula, $\mathrm{X}$ is the number of passengers arriving at zone A within time Ta, $X^{\prime}$ is the passengers leaving zone A within time $\mathrm{Ta}^{\prime}$. According to the data provided and the data found in the official website, I set the average arrival rate or the security check point is 13 person/minute, the arrival rate of pre-check channel is 6 person/minute, the arrival rate of regular channel is 7 person/minute. From the column C, D of the data provided, it works out that the average service time of zone $\mathrm{A}$ is 5.4 person/minute.

Considering the Chicago O'Hare airport, in its official website, the annual average passenger traffic is 77.96 million, and the security facilities in airport have 4 terminal, 11 security check point. Based on the data provided, it assumes that there are two pre-check channels and three regular check channels in zone A, one pre-check channel and three regular check channels or two pre-check and six regular check channels in B zone. After calculation, it can find that in the first situation of zone $\mathrm{B}$, the service rate is greater than the arrival rate, resulting in unlimited queuing, so it can exclude the situation.

When $\rho_{l}=\frac{\rho}{l}=\frac{\lambda}{l \rho}<1$ :

$$
\begin{gathered}
P_{n}=\left\{\begin{array}{l}
\frac{\rho^{n}}{n 1} P_{0}, \quad n=1,2, \cdots l \\
\frac{\rho^{n}}{l ! l^{n-l}} P_{0}, \quad n=l, l+1, \cdots
\end{array}\right. \\
P_{0}=\left(\begin{array}{l}
\left.\sum_{n=0}^{l-1} \frac{\rho^{n}}{n !}+\frac{\rho^{l}}{l !\left(1-\rho_{l}\right)}\right)
\end{array}\right.
\end{gathered}
$$

Under normal circumstances, in the security screening system under stable conditions, when the number of passengers greater than the number of security check desks, the probability that passengers need to wait can be solved by Erlang waiting formula ${ }^{[3]}$ :

$$
\sum_{n=l}^{\infty} P_{n}=\frac{\rho^{l}}{l !\left(1-\rho_{l}\right)} p_{0}
$$

The other model indicators are:

$$
L_{q}=\frac{p_{0} \rho^{l} p_{l}}{l !\left(1-\rho_{l}\right)^{2}}
$$

$L_{s}=$ average line length + the number or passengers who are accepting service:

$$
\begin{aligned}
& L_{s}=L_{q}+\rho \\
& W_{s}=\frac{L_{s}}{\lambda} \\
& W_{q}=W_{s}-\frac{1}{\mu}
\end{aligned}
$$

\section{Conclusions}

The indicator of queuing model in the zone $\mathrm{A}$ and the number of the passengers queuing in zone $\mathrm{A}$ can be calculated. Meanwhile, the service rate in zone A is the arrival rate in zone $\mathrm{B}, l \cdot \mu_{\mathrm{A}}=\lambda_{\mathrm{B}}$. According to the data given, about $56 \%$ of the passengers enter the pre-check channel for security screening in the zone B. Therefore, the pre-check arrival rate of zone B is $56 \% \cdot \lambda_{\mathrm{B}}$. From the data, it can calculate that the average time a passenger passing the millimeter wave body scanner is $11.6 \mathrm{~s}$, the average time a passenger passing the X-ray is $7 \mathrm{~s}$. After calculating the queuing model of pre-check passengers in the zone B, similarly, according to the regular passenger arrival rate $44 \%$. 
$\lambda_{\mathrm{B}}$ in the zone $\mathrm{B}$, calculate the indicators in the queuing theory of regular passengers. Then, let passengers who have finished the security screening in zone $\mathrm{B}$ enter the zone $\mathrm{D}$ at a certain proportion. Here, model simplifies the security screening time of zone D, makes the security screening time of zone $\mathrm{D}$ the fixed value. The other passengers all enter the zone $\mathrm{C}$, and the number of channels in zone $\mathrm{C}$ is the same as zone $\mathrm{B}$.

\section{Acknowledgments}

My deepest gratitude goes first and foremost to Teacher $\mathrm{Gu}$, our supervisor, for her constant encouragement and guidance. She has walked me through all the stages of the writing of this thesis. Without her consistent and illuminating instruction, this thesis could not have reached its present form. Second, I would like to express my heartfelt gratitude to Qihan Liu, my roommate, who led me into the world of data. I also owe my sincere gratitude to my friends and my fellow classmates who gave me their help and time in listening to me and helping me work out my problems during the difficult course of the thesis.

\section{References}

[1] Yildiz, Y. O., Abraham, D. Q., Panetta, K., \&Agaian, S. (2008). A new concept of airport security screening. In Proceeding of the IEEE Conference on Technologies for Homeland Security (pp. 444-448), May 12-13, 2008, Waltham, Washington.

[2] Designing production and service systems using queuing theory: principles and application to an airport passenger security screening system[J]. International Journal of Services \& Operations Management, 2010, 6(2):206-225.

[3] R. T. Wang, “Title of Chapter,” in Classic Physiques, edited by R. B. Hamil (Publisher Name, Publisher City, 1999), pp. 212-213. 